\title{
Generation and Focusing of Orbital Angular Momentum Based on Polarized Reflectarray at Microwave Frequency
}

\author{
Fengxia $\mathrm{Li}^{\circledR}$, Haiyan Chen ${ }^{\circledR}$, Yang Zhou ${ }^{\circledR}$, Jianwei You ${ }^{\circledR}$, Member, IEEE, Nicolae C. Panoiu ${ }^{\circledR}$, Member, IEEE, \\ Peiheng Zhou, Member, IEEE, and Longjiang Deng
}

\begin{abstract}
A novel polarized reflectarray is designed, fabricated, and experimentally characterized to show its flexibility and efficiency to control wave generation and focusing of orbital angular momentum (OAM) vortices with desirable OAM modes in the microwave frequency regime. In order to rigorously study the generation and focusing of OAM, a versatile analytical theory is proposed to theoretically study the compensation phase of reflectarray. Two prototypes of microwave reflectarrays are fabricated and experimentally characterized at 12 GHz: one for generation and one for focusing of OAM-carrying beams. Compared with the OAM-generating reflectarray, the reflectarray for focusing OAM vortex can significantly reduce the beam diameter, and this can further improve the transmission efficiency of the OAM vortex beams. We also show that the numerical and experimental results agree very well. The proposed design method and reflectarrays may spur the development of new efficient approaches to generate and focus OAM vortex waves for applications to microwave wireless communications.
\end{abstract}

Index Terms-Microwave, orbital angular momentum (OAM) generation, OAM focusing, polarized reflectarray.

Manuscript received August 3, 2020; revised September 30, 2020 and October 26, 2020; accepted October 31,2020. This work was supported in part by the National Natural Science Foundation of China under Grant 51972046 and Grant 51772042; in part by "111" Center under Grant B13042; in part by China Scholarship Council (CSC); and in part by the European Research Council under Grant ERC-2014-CoG-648328. (Corresponding author: Haiyan Chen.)

Fengxia Li is with the Key Laboratory of Multi-Spectral Absorbing Materials and Structures of Ministry of Education, National Engineering Research Center of Electromagnetic Radiation Control Materials, University of Electronic Science and Technology of China, Chengdu 610054, China, also with the State Key Laboratory of Electronic Thin Film and Integrated Device, University of Electronic Science and Technology of China, Chengdu 610054, China, and also with the Department of Electronic and Electrical Engineering, University College London, London WC1E 7JE, U.K. (e-mail: 1fx05191fx@163.com).

Haiyan Chen, Yang Zhou, Peiheng Zhou, and Longjiang Deng are with the Key Laboratory of Multi-Spectral Absorbing Materials and Structures of Ministry of Education, National Engineering Research Center of Electromagnetic Radiation Control Materials, University of Electronic Science and Technology of China, Chengdu 610054, China, and also with the State Key Laboratory of Electronic Thin Film and Integrated Device, University of Electronic Science and Technology of China, Chengdu 610054, China (e-mail: chenhy@uestc.edu.cn; zhouyaug@163.com; zhouph1981@163.com; denglj@uestc.edu.cn).

Jianwei You and Nicolae C. Panoiu are with the Department of Electronic and Electrical Engineering, University College London, London WC1E 7JE, U.K. (e-mail: j.you@ucl.ac.uk; n.panoiu@ucl.ac.uk).

Color versions of one or more figures in this article are available at https://doi.org/10.1109/TMTT.2020.3040449.

Digital Object Identifier 10.1109/TMTT.2020.3040449

\section{INTRODUCTION}

W ITH the rapid development of wireless communication technology, it has become imperative to improve the system capacity and spectral efficiency to meet the exponential growth of demand in the area of broadband data transfer, data centers, and cloud-based services. To overcome this challenge, orbital angular momentum (OAM) has emerged as an effective means to carry information due to its multiple orthogonal modes can simultaneously transfer at the same frequency in a single communication channel, offering alternative and flexible degrees of freedom [1] without increasing the frequency bandwidth [2]-[4]. As a result, the use of OAM in wireless communications has become a field of intense research, due to its potential applications, especially in the microwave regime [5]-[8]. Therefore, it is very important to develop effective methods to generate OAM vortex waves at microwave frequency. Within the broad array of applications of OAM, how to efficiently generate, manipulate, and receive OAM-carrying beams are the most important issues. Recently, versatile antennas and antenna arrays have been demonstrated to generate OAM beams, the underlying principle being the introduction of an azimuthal phase factor in order to generate single or multiple OAM modes [9]-[11]. However, such devices have some inherent limitations. Thus, in practical applications, antennas based on spiral reflectors have large thicknesses and are usually difficult to fabricate [12], [13]. Furthermore, to generate OAM using antenna arrays, one needs a complex feeding network, so as to generate a phase difference between array elements [14]-[16]. This can increase the complexity of system integration and production costs. However, instead of the above designs, reflectarrays are gradually considered to be one of the practical ways for generating vortex waves in the radio frequency domain. Reflectarray can be designed to produce single or multiple beams using single- or multiple-layer configurations at different frequency bands [17]-[20]. A single-layer, dual-frequency unit for generating OAM in the microwave range for multifunctional OAM with required OAM mode, beam number, and direction was reported in [21]. According to [22], a high-efficiency planar reflectarray with a small size was proposed to effectively convert arbitrarily polarized waves to OAM waves. Furthermore, Jiang et al. [23] designed a class of low-profile and broadband dual-circularly polarized reflectarrays with 
independent beamforming for circularly polarized waves with opposite handedness. Meanwhile, a dual-band dual-polarized reflectarray for generating dual beams with respect to carrying two different OAM topological charges operating in the $C$-band in horizontal polarization and the $X$-band in vertical polarization was proposed in [24]. They mainly concern about high efficiency and high OAM mode purity in a low-profile configuration. More recently, metasurfaces based on catenary and other structures have also been reported to generate vortex waves successfully, providing another novel route to achieve OAM beam.

Metasurfaces have been demonstrated to have enhanced ability to facilitate OAM generation and processing [25]-[27]. As the 2-D equivalent of metamaterials, metasurfaces have been widely used to manipulate electromagnetic (EM) waves [28]-[32], chiefly due to their ultrathin and subwavelength characteristics. In particular, due to their remarkable abilities to manipulate the amplitude, phase, and polarization state of EM waves [33]-[37], it is possible to employ metasurfaces to generate vortex beams with an arbitrary topological number, as it has been confirmed by a series of experiments in the optical and microwave regime [38]-[43]. Yu et al. [44] demonstrated a reflective metasurface to generate OAM in the radio frequency domain at a single frequency point, but the bandwidth is narrow and the size is relatively large. Metasurfaces composed of Pancharatnam-Berry (PB) phase elements are demonstrated to control the conical beam generation by Ding et al. [45]; however, it did not consider the issue of transmission and efficiency. Apart from this, OAM vortex waves have annular structure and are divergent, and thus, the inner diameter of the beam is a key factor that determines their suitability for communications based on specific OAM modes. In addition, as the order of the OAM mode and the propagation distance increase, the degree of beam divergence increases too. Therefore, it is particularly important to develop effective methods for focusing on OAM vortex waves.

In the microwave regime, despite that previous research focused on OAM beam generation, it has not been demonstrated that a reflectarray based on reflective polarized converter can be used to generate and focus OAM beams simultaneously. Recently, the control of polarization states of EM waves based on periodic structure has attracted intense research interest at the frontier of science and engineering due to the ability to modify the amplitude and phase of EM waves [46]-[48]. By controlling the phase and amplitude responses of the unit cells in reflectarray, a vortex beam with arbitrary topological charge can be easily realized. This has spurred great interest in designing polarized reflectarrays to generate and focus OAM beams. In this article, we propose two types of polarized reflectarrays to generate and focus OAM beams at microwave frequencies. The cross-polarization conversion efficiency of these polarized elements is over $70 \%$ in the bandwidth above 55\%, and these unit cells can achieve $2 \pi$ phase variation. These polarized reflectarrays provide great flexibility in phase control while greatly suppressing the transmission loss. Moreover, a theoretical formula for the phase distribution is derived and used to design the two reflectarrays for the generation and focusing OAM beams. These reflective polarized reflectarrays have the advantages of being ultrathin and lightweight and provide great flexibility in tailoring the phase distribution in its unit cell. Two of these reflectarrays were designed, fabricated, and measured at $12 \mathrm{GHz}$, so as to validate the theoretical predictions, and generation and focusing of OAM vortex waves is achieved by using quasi-spherical beams and we found that the results of numerical simulations agree very well with the experimental measurements. Thus, the simulations and measurements demonstrate that the generation and focusing of OAM-carrying beams can be flexibly achieved by using polarized reflectarrays. However, considering the communication requirements, it can be found that it is necessary to further increase the transmission and focusing distance of the OAM beam, which will also be one of our future research topics.

\section{Reflectarrays Design AND Results}

A reflective polarized converter based on a single-layer unit cell is chosen here. The proposed element is subwavelength, and its parameters are shown in Fig. 1(a). The square split ring and the metal ground plane are separated by a Teflon dielectric spacer, and the reflection phase varies with the change of parameters $b$ and $w$. The unit cell period $p=10 \mathrm{~mm}$, thickness of substrate $h=3 \mathrm{~mm}$ with relative permittivity $\varepsilon_{r}=2.65$, and the other geometrical parameters of the element are: $a=6 \mathrm{~mm}, a_{1}=5 \mathrm{~mm}$, and $b_{1}=3 \mathrm{~mm}$. The values of parameters and reflection phase of the element are listed in Table I.

Under linearly polarized incidence, the cross-polarization reflective coefficient is above $70 \%$ with the bandwidth of more than 55\%, as shown in Fig. 2(a). In addition, the results plotted in Fig. 2(b) demonstrate that the phase of the reflective cross-polarized components can change by $360^{\circ}$ by varying the values of $b$ and $w$, which is enough to produce OAM vortex waves with arbitrary topological number.

The proposed reflectarray consists of polarized elements, metallic ground plate, and an illuminating feed antenna, as shown in Fig. 1(c). Considering a reflectarray, which consists of $M * N$ elements that are illuminated by a feed source located at the position of $r_{d}, r_{\mathrm{mn}}$ is the distance between the $m n$th element and the coordinate center, and the generating OAM vortex waves propagate along the $z$-axis.

\section{ANALYSis AND DiscusSiON}

\section{A. Numerical Results}

According to the Helmholtz equation in free space [49], for vortex waves that propagate along the $z$-axis, the vector electric field $\boldsymbol{E}_{l}$ in cylindrical coordinates can be expressed as follows:

$$
\boldsymbol{E}_{\boldsymbol{l}}(\boldsymbol{r}, \varphi, z)=\boldsymbol{E}_{\mathbf{0}} \exp (i l \varphi) \exp \left(-i k_{0} z\right)
$$

where $l$ is an arbitrary integer representing the topological charge and is also the OAM mode number. We assumed that if the coordinate of a unit cell is $(x, y)$, then $\varphi=\arctan (y / x)$, which is related to the origin point, $k_{0}$ is the wavenumber of free spaces, and $\boldsymbol{E}_{0}$ is a constant vector. As shown in Fig. 1(c), 


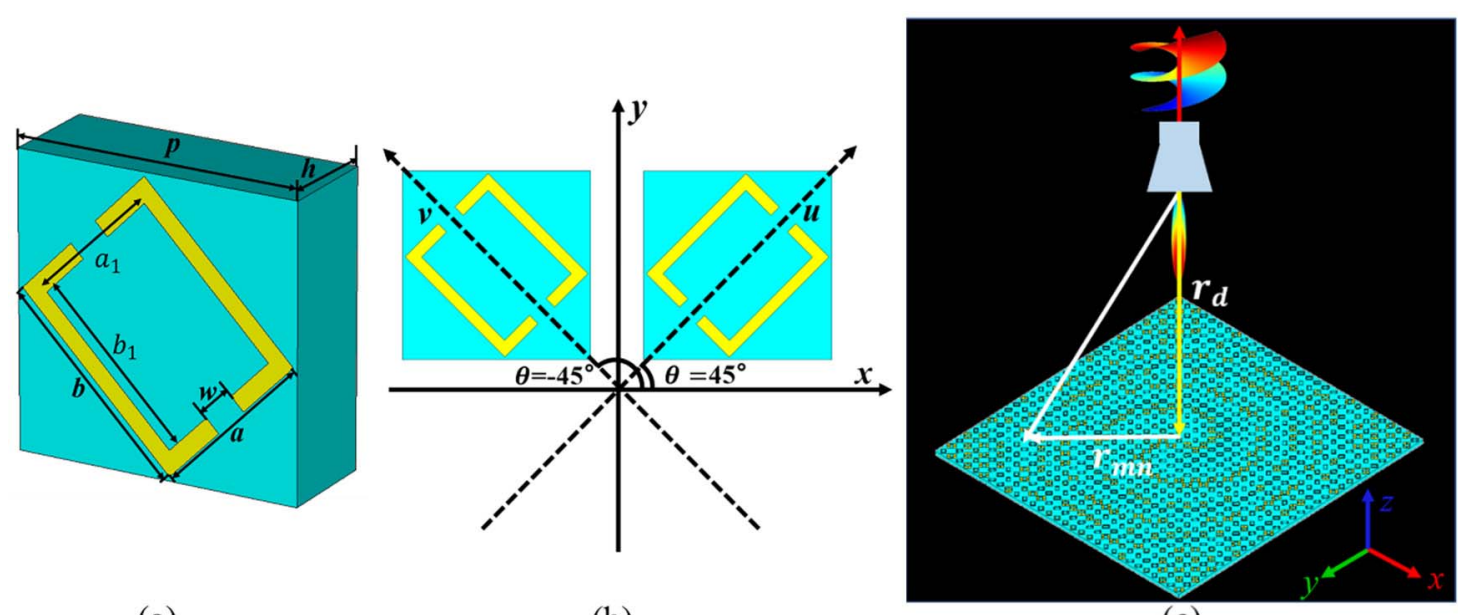

(a)

(b)

(c)

Fig. 1. (a) Schematic of the reflective polarized converter with variable $w$ and $b$. (b) Polarization conversion structures of $45^{\circ}$ symmetrical and $-45^{\circ}$ symmetrical, and $\theta$ is the angle between the $x$-axis and the $u / v$ symmetry axis of the structure. (c) Configuration of OAM-generation reflectarray.

TABLE I

Parameters of SubwaVelength Elements

\begin{tabular}{|c|c|c|c|c|c|c|c|c|}
\hline Parameters & Element 1 & Element2 & Element3 & Element4 & Element5 & Element6 & Element7 & Element8 \\
\hline $\mathrm{b} / \mathrm{mm}$ & 3.6 & 7.8 & 5.0 & 4.4 & 3.6 & 7.8 & 5.0 & 4.4 \\
\hline$w / \mathrm{mm}$ & 0.8 & 0.2 & 0.2 & 1.0 & 0.8 & 0.2 & 0.2 & 1.0 \\
\hline$\theta /{ }^{\circ}$ & -45 & 45 & 45 & 45 & 45 & -45 & -45 & -45 \\
\hline Unit cells & & & & & & & & \\
\hline \multicolumn{9}{|l|}{ Reflection } \\
\hline Phase ${ }^{\circ}$ & -172 & -116 & -74 & -32 & 9 & -296 & -255 & -212 \\
\hline
\end{tabular}

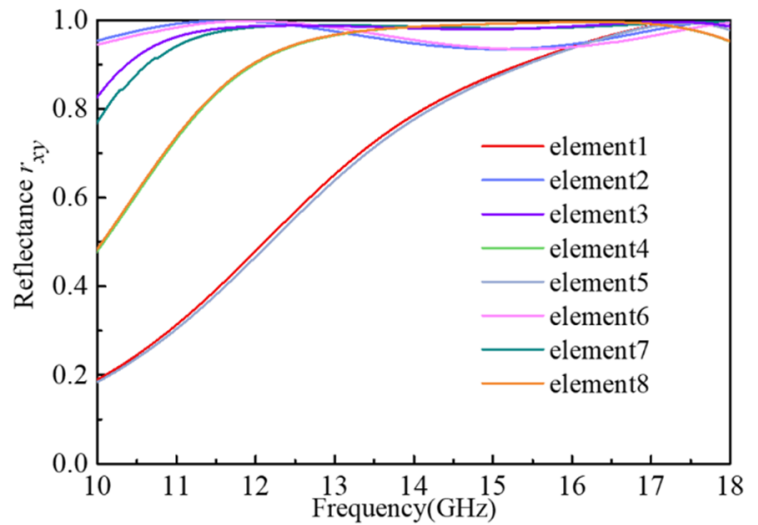

(a)

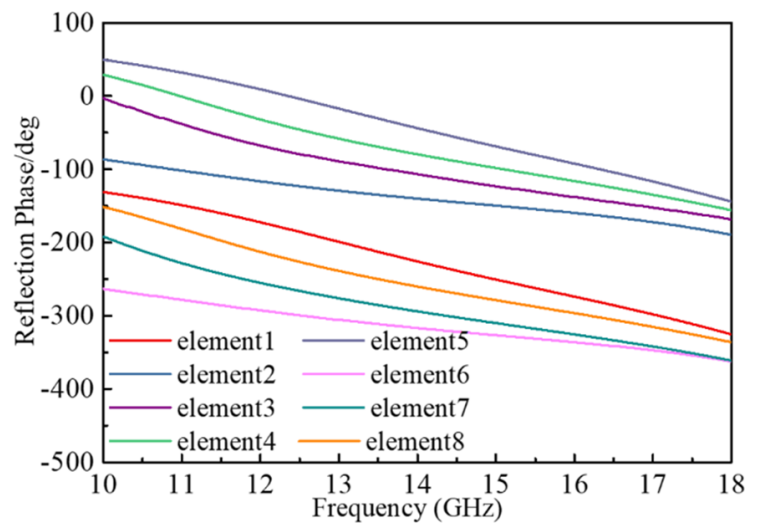

(b)

Fig. 2. (a) Simulation of cross-polarization reflection coefficient of the polarized unit cells at $12 \mathrm{GHz}$ versus frequency with the change of parameters $b$ and $w$. (b) Simulated reflection phase of the polarized unit cells at $12 \mathrm{GHz}$ versus frequency with the change of parameters $b$ and $w$.

we can get the expression of an input phase of the standard horn, that is

$$
\varphi_{I}=k_{0} \sqrt{x^{2}+y^{2}+r_{d}^{2}}
$$

Then, the compensating phase required at each polarized reflective unit cell in the desired direction can be obtained by

$$
\varphi_{m n}=l \arctan (y / x)-k_{0} \sqrt{x^{2}+y^{2}+r_{d}^{2}}
$$




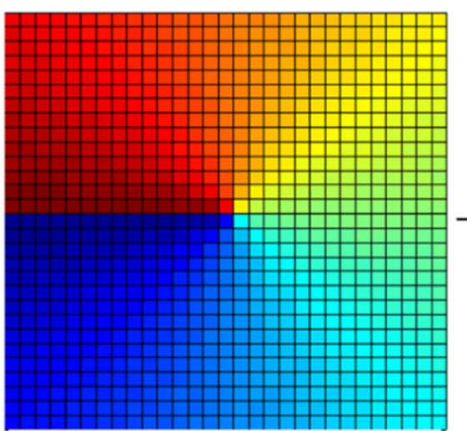

(a)

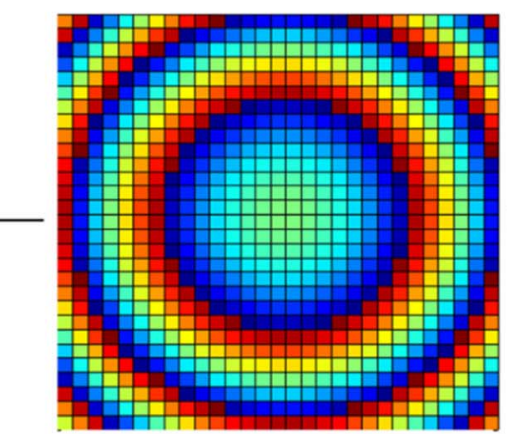

(b)

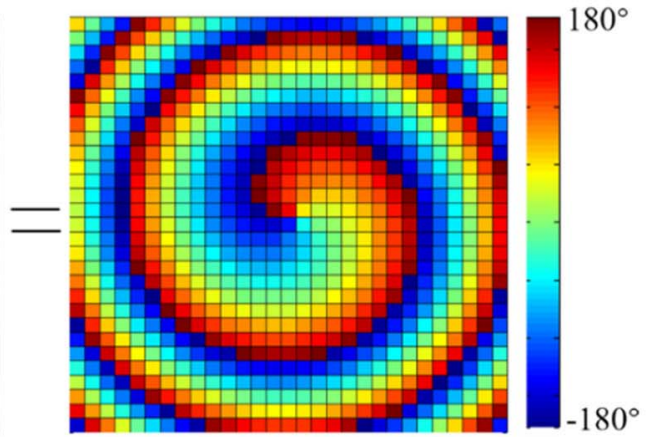

(c)

Fig. 3. Calculation process and phase distribution compensated on the reflectarray based on the polarized reflective elements. (a) Output phase of vortex wave. (b) Input phase of incident source. (c) Compensate phase of reflectarray.

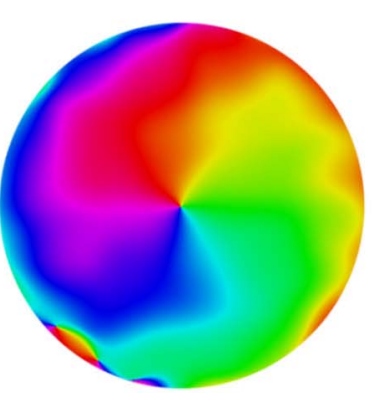

(a)

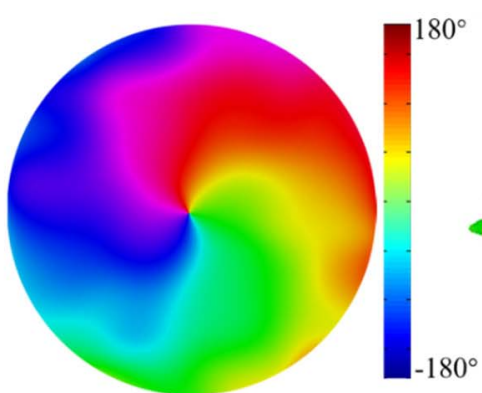

(b)

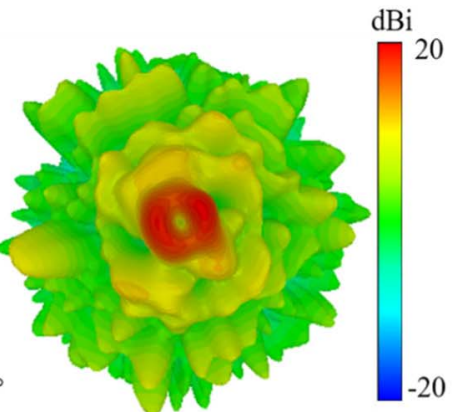

(c)

Fig. 4. Simulation results of generating OAM vortex waves with topological charge $l=1$ at 12 GHz. (a) Phase distribution of electrical field at cross sections $z=600 \mathrm{~mm}\left(z=24 \lambda_{0}\right)$ in the $x y$ plane. (b) Phase distribution of electrical field at cross sections $z=800 \mathrm{~mm}\left(z=32 \lambda_{0}\right)$ in the $x y$ plane. (c) Top view of simulated radiation pattern in the far-field zone.

where $\varphi_{\mathrm{mn}}$ is the azimuthal angle of the $m n^{\text {th }}$ element and $r_{d}$ is the distance between the horn and the reflectarray. Based on the phase distribution of the required function of the reflectarray, the specific unit cell at any location can be designed.

The numerical results shown in Fig. 3 reveal that the phase variation of the reflectarray must cover $360^{\circ}$. The output phase of the vortex wave and the input phase of the incident source are shown in Fig. 3(a) and (b), respectively. The necessary compensating phase generated by the reflectarray can be calculated by using the EM field superposition principle as given in (3), and it is given in Fig. 3(c). Theoretically, OAM vortex waves with arbitrary topological charges can be generated in terms of the continuous spatial phase change by employing reflectarrays with periodic elements.

\section{B. Simulation Results}

For illustration, we designed a reflectarray using polygonal elements to generate OAM beams. The reflectarray contained $30 \times 30$ elements based on these eight basic unit cells presented in Table I. The layout is a square array with the dimension of $300 \mathrm{~mm} \times 300 \mathrm{~mm}$, as shown in Fig. 1(c). The topological charge of OAM-generation design was chosen to be 1 . In order to verify the performance of the designed

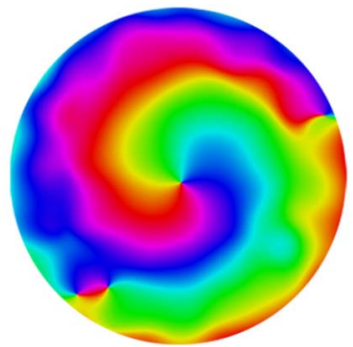

(a) (b)

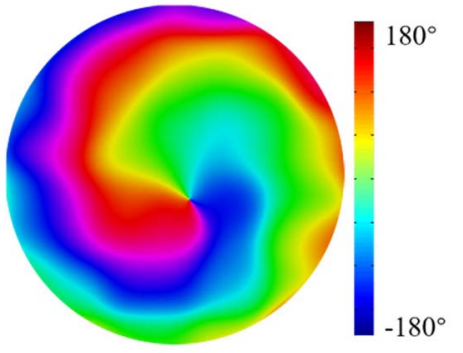

Fig. 5. Simulation phase distributions of electrical field for OAM-focusing vortex beam with topological charge $l=1$ at $12 \mathrm{GHz}$ in the $x y$ plane. (a) $z=$ $600 \mathrm{~mm}$. (b) $z=800 \mathrm{~mm}$.

reflectarray, numerical simulations are performed using the CST Microwave Studio for all these schemes with normal incident wave and polarization along the $y$-axis, and full-wave simulations based on the finite-difference time-domain (FDTD) technique have been performed at $12 \mathrm{GHz}\left(\lambda_{0}=25 \mathrm{~mm}\right)$, to analyze the characteristics of OAM-generation by the reflectarray. The unit cell boundary conditions were applied along the $x$-/y-directions. A horn antenna was used as the feeding source at normal incidence, and the feed point was located in front of the reflectarray 


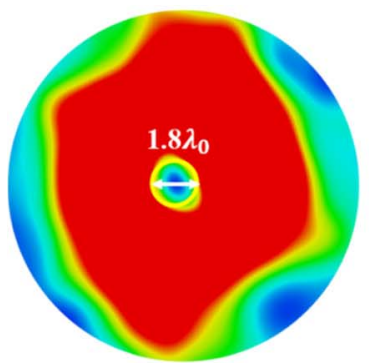

(a)

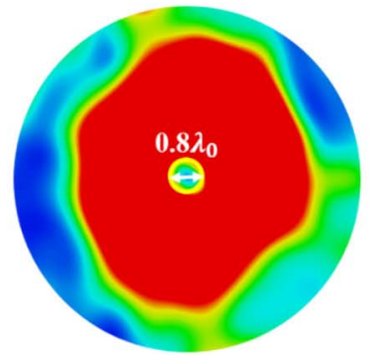

(d)

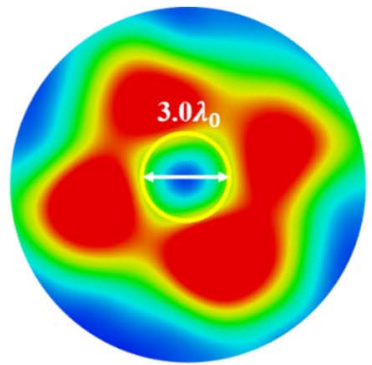

(b)

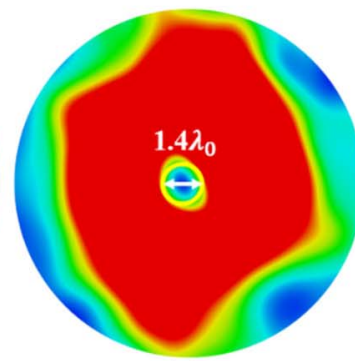

(e)

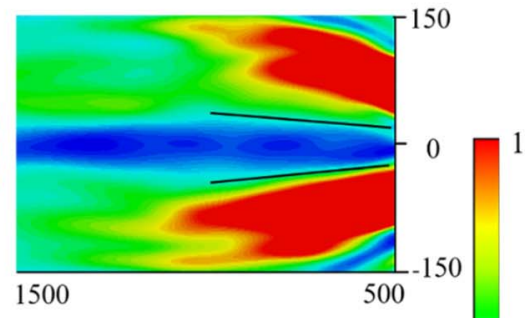

(c)

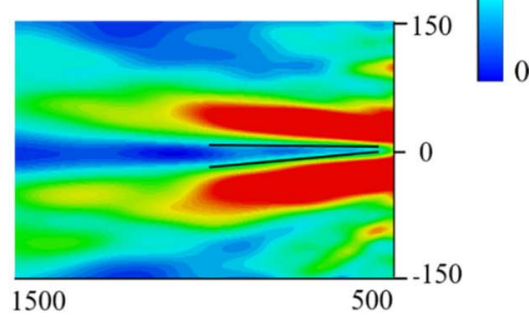

(f)

Fig. 6. Simulation results of electrical field energy of polarized reflectarrays for generating and focusing vortex beams with a topological charge of 1 at $12 \mathrm{GHz}$. (a) Simulated distribution of energy in the $x y$ plane for OAM-generating reflectarray at cross sections $z=600 \mathrm{~mm}$. (b) Simulated distribution of energy in the $x y$ plane for OAM-generating reflectarray at cross sections $z=800 \mathrm{~mm}$. (c) Simulated distribution of energy for OAM-generating beams in the $y z$ plane. (d) Simulated distribution of energy in the $x y$ plane for OAM-focusing reflectarray at cross sections $z=600 \mathrm{~mm}$. (e) Simulated distribution of energy in the $x y$ plane for OAM-focusing reflectarray at cross sections $z=800 \mathrm{~mm}$. (f) Simulated distribution of energy in the $y z$ plane for OAM-focusing beams.

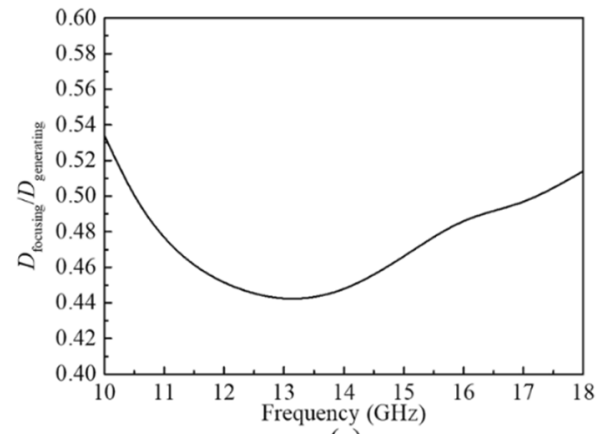

(a)

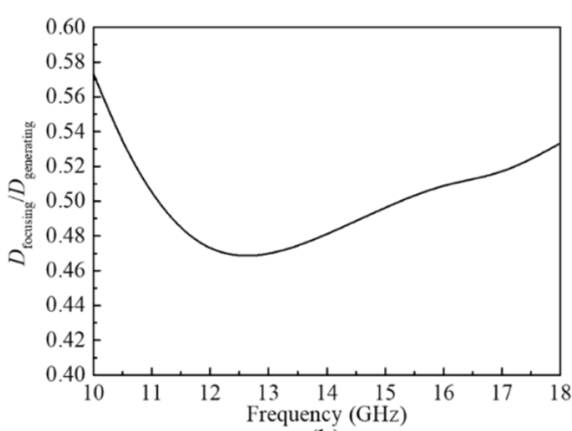

(b)

Fig. 7. Simulation beam diameters ratio of polarized reflectarray between OAM-focusing and generation vortex beams with a topological charge of 1 versus frequency. (a) $z=600 \mathrm{~mm}$. (b) $z=800 \mathrm{~mm}$.

at $10 \lambda_{0}$ distance away from the reflectarray along the $z$-axis. Fig. 4(a) and (b) shows the simulated phase distribution at the cross sections $z=600 \mathrm{~mm}\left(z=24 \lambda_{0}\right)$ and $800 \mathrm{~mm}(z=$ $32 \lambda_{0}$ ), which are perpendicular to the propagation direction, the characteristics of the vortex waves can be seen from the phase distribution. Fig. 6(a) and (b) shows the simulated distribution of energy at the cross sections $z=600$ and $800 \mathrm{~mm}$, the inner diameters $(D)$ of the energy distribution in the $x y$ plane are $1.8 \lambda_{0}$ for $z=24 \lambda_{0}$ and $3.0 \lambda_{0}$ for $z=$ $32 \lambda_{0}$, respectively. From these results, it can be clearly seen that the inner diameters of the energy distribution along the propagation direction increase when the distance increases. The corresponding simulation of the 3-D scattering patterns in the far-field zone is shown in Fig. 4(c), and the results reveal that there is an amplitude null in the center of the beam, which agrees with the properties of OAM vortex beams with a topological charge equal to 1. Fig. 6(c) shows the simulated distribution of energy in the $y z$ plane. It can be seen in this figure that, as expected, when the propagation distance increases, the radius of the beam increases, indicating that the vortex beam with OAM mode diverges due to diffraction.

As shown in Fig. 6(a) and (b), OAM vortex waves would expand along the propagation direction, which creates serious problems at the receiver end in communication systems. Therefore, it is necessary to reduce the radius of the vortex beam along the propagation path. In order to achieve this, the phase function of the reflectarray should be equivalent to the interference pattern between a focusing phase factor and a spiral phase plate so that the cross-polarized reflective converter can constructively focus OAM beams with arbitrary topological charge. This can be fulfilled by the superposition of the spiral phase profile and focusing phase factor [50]. The required compensation phase $\varphi_{\mathrm{mn}}^{c}$ of a unit cell located at the 
position of $(x, y)$ can be expressed as

$$
\begin{array}{r}
\varphi_{m n}^{c}=l \arctan (y / x)+k_{0}\left(\sqrt{x^{2}+y^{2}+f_{1}^{2}}-\left|f_{1}\right|\right) \\
-k_{0} \sqrt{x^{2}+y^{2}+r_{d}^{2}}
\end{array}
$$

where $f_{1}$ represents the focal length.

Simulations were performed at $12 \mathrm{GHz}\left(\lambda_{0}=25 \mathrm{~mm}\right)$, and the topological charge of OAM-focusing design was chosen to be 1. The parameters of these eight unit cells are the same as those of the OAM-generation design. This focusing reflectarray, which is composed of $30 \times 30$ unit cells, has a focal length of $24 \lambda_{0}\left(f_{1}=600 \mathrm{~mm}\right)$. Fig. 5 shows the simulation results of phase distribution at different propagation distances in the $x y$ plane at 600 and $800 \mathrm{~mm}$. It can be seen from these figures that, when compared to the phase at the same position corresponding to the OAM-generating reflectarray, the diameters of the vortex wave are reduced and the divergence of OAM beams is suppressed. In Fig. 6, which shows the spatial profile of the energy of the beam, it can be clearly observed that doughnut-shaped energy intensity patterns were obtained and that the energy distribution approaches the center axis during propagation when compared with the OAM-generating reflectarray at cross sections $z=600$ and $800 \mathrm{~mm}$, which can be seen in Fig. 6(d) and (e). The inner diameters of energy map of OAM-generation reflectarray are $1.8 \lambda_{0}$ and $3.0 \lambda_{0}$ for $z=24 \lambda_{0}$ and $32 \lambda_{0}$ in the $x y$ plane, whereas the inner diameters of energy map of OAM-focusing reflectarray are $0.8 \lambda_{0}$ and $1.4 \lambda_{0}$, respectively. At the same propagation position, the diameters of OAM-focusing beam are reduced and the OAM-focusing reflectarray is obviously more constrictive, whereas the diameter of energy ring is about half of that OAM-generation reflectarray in the position of $24 \lambda_{0}$ and $32 \lambda_{0}$ which demonstrates the efficiency of the OAM-focusing of this reflectarray.

In Fig. 6(c) and (f), we present the simulated results of OAM-generating and focusing vortex beams in the $y z$ plane. From these figures, one can see that as the propagation distance increases, the radius of the OAM-focusing beams is reduced when compared to that of the OAM-generating beam, which indicates that the OAM-focusing reflectarray can efficiently arrest deleterious diffractive effects.

As is indicated by the above discussions, further numerical simulations reveal that the OAM-focusing reflectarray is obviously more constrictive, which can be seen in Fig. 7. As shown in Fig. 7(a) and (b), the diameter of the OAM-focusing reflectarray is smaller than that of the OAM-generation reflective reflectarray at the distance of 600 and $800 \mathrm{~mm}$, respectively. Also, the frequency points with uniform phase difference and larger cross-polarization conversion amplitude have a better focusing effect.

\section{FABRICATION AND EXPERIMENTAL RESULTS}

To experimentally validate the design principle, theoretical method, and simulation results, the prototypes of the two OAM-generation and focusing reflectarrays that are shown in Fig. 8(b) and (c) contain $30 \times 30$ unit cells and have an overall size of $300 \mathrm{~mm} \times 300 \mathrm{~mm}$, which were fabricated

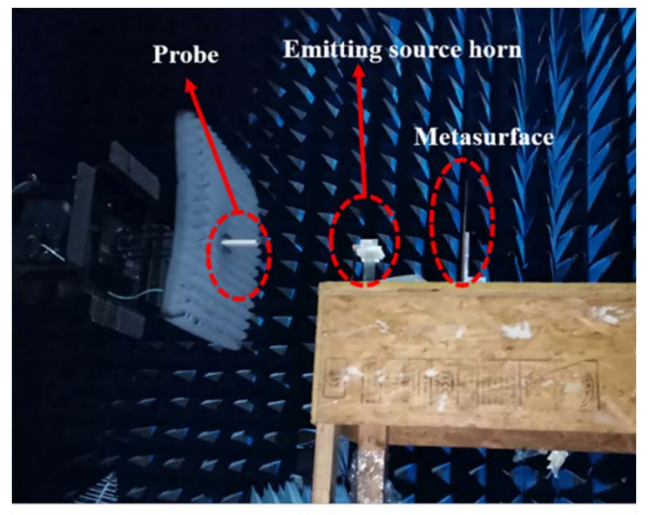

(a)

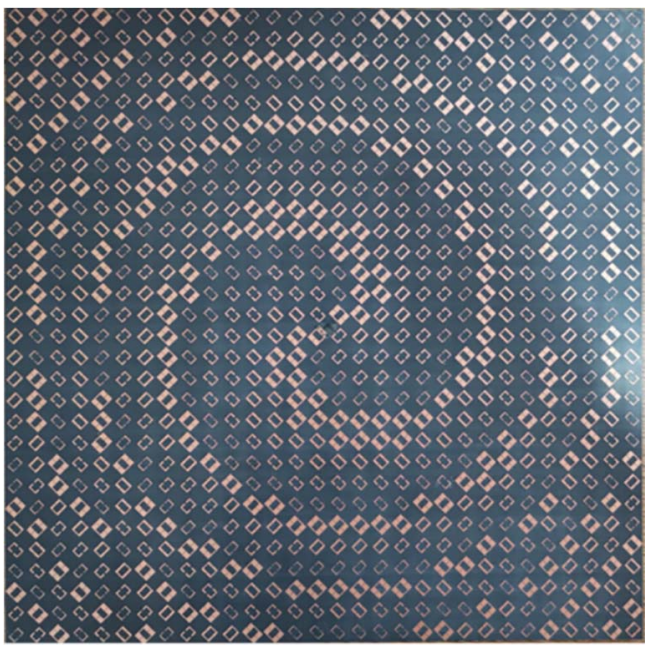

(b)

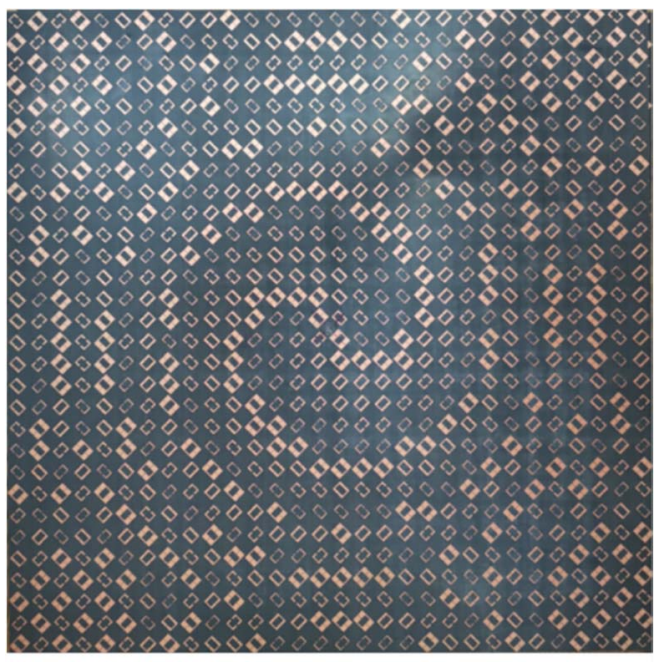

(c)

Fig. 8. (a) Experimental system configuration for the OAM vortex waves measurement in the $x y$ plane. (b) Fabricated prototype of the OAM-generation reflectarray. (c) Fabricated prototype of the OAM-focusing reflectarray.

using low-cost printed circuit board (PCB) technique and measured in an anechoic chamber, as shown in Fig. 8. The near-field planar scanning technique was applied to characterize the OAM vortex waves. The experimental system is shown in Fig. 8(a). We measured the scattering patterns of the samples in a standard microwave anechoic chamber, and 


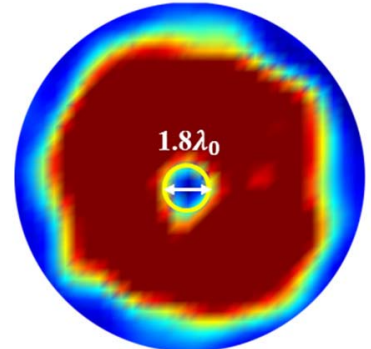

(a)

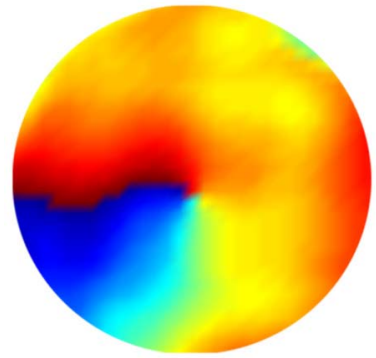

(c)

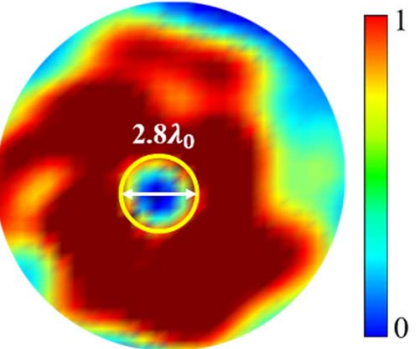

(b)

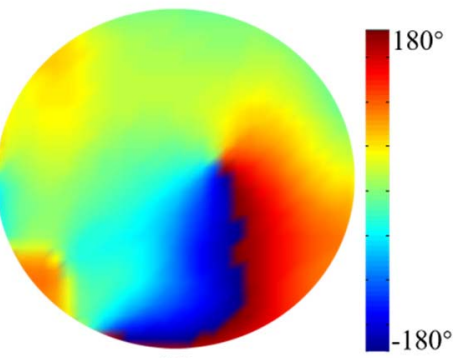

(d)
Fig. 9. Experimental results of OAM-generation vortex waves with topological charge $l=1$ at $12 \mathrm{GHz}$ in the $x y$ plane. (a) Energy distribution of electrical field at cross sections $z=600 \mathrm{~mm}$. (b) Energy distribution of electrical field at cross sections $z=800 \mathrm{~mm}$. (c) Phase distribution of electrical field at cross sections $z=600 \mathrm{~mm}$. (d) Phase distribution of electrical field at cross sections $z=800 \mathrm{~mm}$.

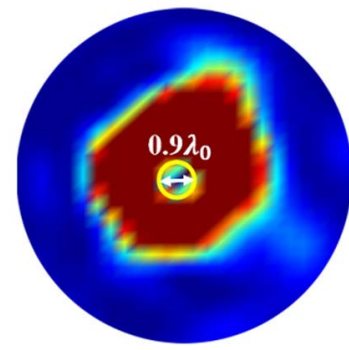

(a)

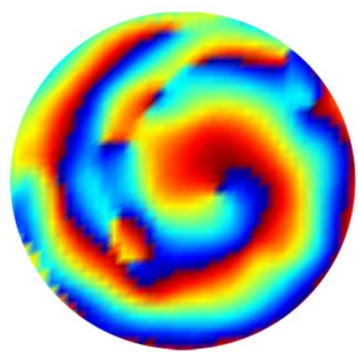

(c)

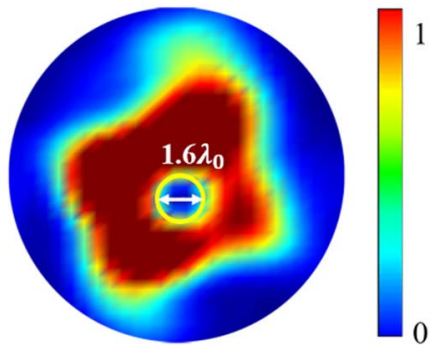

(b)

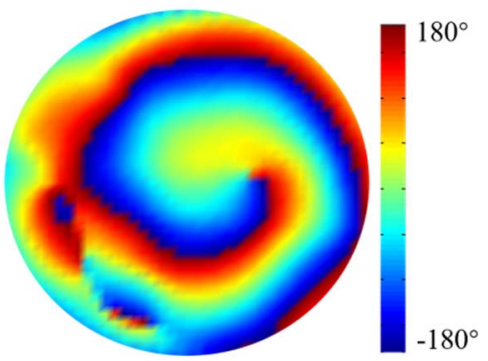

(d)
Fig. 10. Experimental results of the polarized reflectarray for OAM-focusing vortex beam with topological charge $l=1$ in the $x y$ plane. (a) Energy distribution of electrical field at cross sections $z=600 \mathrm{~mm}$. (b) Energy distribution of electrical field at cross sections $z=800 \mathrm{~mm}$. (c) Phase distribution of electrical field at cross sections $z=600 \mathrm{~mm}$. (d) Phase distribution of electrical field at cross sections $z=800 \mathrm{~mm}$.

the measured results are calibrated to the same size metal plate. These measurements were performed at the frequency of $12 \mathrm{GHz}\left(\lambda_{0}=25 \mathrm{~mm}\right)$ in the $x y$ plane in order to get the electric field intensities and phase distributions. In the measurement system, a wideband horn antenna (80180HA20N) is employed as the excitation at a distance of $d=250 \mathrm{~mm}$ $\left(10 \lambda_{0}\right)$ away from the center of the reflectarrays. An active antenna (120WOEWPN) is used as a field probe and is capable to measure the cross-polarization component of the reflected electric field through the network analyzer (8720ET). The horn antenna and probe were connected with the network analyzer by the coaxial cable to measure the sample. The probe shown in Fig. 8(a) is used as the receiving end whose position is controlled by a motion controller. The orientation of the probe antenna here is set to be vertical to the samples in order to obtain the components of the electric fields. In order to observe the stability of the vortex wave as it propagates, the scanning plane is set to be 600 and $800 \mathrm{~mm}$ from the samples. A small scanning step of $1 \mathrm{~mm}$ is selected to acquire intensities and phase distributions at the sampling planes. With the variation of the position of the field probe via the motion controller, the $x y$ plane can be covered and the experimental field intensities and phase profiles can be measured.

The measured energy intensity and phase distribution of the reflected electric field are shown in Figs. 9 and 10 in the $x y$ plane at cross sections $z=600 \mathrm{~mm}$ and $z=800 \mathrm{~mm}$, respectively. Compared with the simulated OAM spatial field distribution (see Figs. 4-6), we can see that the OAM vortex wave of generation and focusing reflectarrays with $l=1$ is observed. The simulated and measured results are in good agreement. There is a subtle difference in the measured energy intensity and phase distribution due to the supporting structure of the feeding horn and the coaxial cable of the reflection system. However, the major features of the spatial phase distribution can be clearly recognized. The typical feature of the field intensity is a perfect doughnut-shaped intensity map, and we can see clearly that there is a null in the center of the beam, which corresponds to the property of vortex waves with OAM. From Figs. 9(a) and (b) and 10(a) and (b), it can be clearly seen that comparing to OAM mode at the same propagation distance, the OAM-focused beam is more radially confined, the radius of energy ring is smaller than the OAM-generation beam, and the measured inner diameters of the energy distribution for OAM-generation vortex waves are $1.8 \lambda_{0}$ and $2.8 \lambda_{0}$ for $z=24 \lambda_{0}$ and $32 \lambda_{0}$ in the $x y$ plane; at the same position, the inner diameters of the energy distribution for OAM-focusing vortex waves are $0.9 \lambda_{0}$ and $1.6 \lambda_{0}$, respectively, which agrees with the simulation results. The phase distributions shown in Figs. 9(c) and (d) and 10(c) and (d) are also in agreement with the simulation results, which validates our theoretical approach.

\section{CONCLUSion}

In summary, we have proposed an approach to generate and focus OAM modes using polarized reflectarrays. These OAM-generation and focusing vortex waves with the same topological charges have been theoretically and experimentally demonstrated in the microwave regime via phase analysis. Thus, based on the superposition of phase profile of spiral phase plate and focusing factor, the OAM-focusing beams can be achieved, their inner radius along the propagation direction being reduced as compared to that of OAM-generating reflectarray, and the OAM-focusing reflectarray is obviously 
more constrictive, while the diameter of energy ring is about half of that OAM-generation reflectarray in the same position. The OAM-generating and focusing prototypes were fabricated and measured to verify the theoretical and numerical predictions. The agreement between the simulation and measurement results confirms the validity of our design procedure. By using the proposed configuration, it is much easier to produce the OAM-focusing vortex waves with different mode numbers, and it also opens a new way to generate and focus OAM vortex waves for microwave wireless communication applications. This proposed method would be particularly useful to design ultrathin reflectarrays for focusing vortex beams that carry desirable OAM modes to potentially improve their communication efficiency.

\section{ACKNOWLEDGMENT}

The work of experimental testing was supported in part by Prof. Shiwei Qu, Dr. Yafei Wu, and Dr. Yunpeng Zhang.

\section{REFERENCES}

[1] R. A. Beth, "Mechanical detection and measurement of the angular momentum of light," Phys. Rev., vol. 50, no. 2, pp. 115-125, Jul. 1936.

[2] Y. Yan et al., "High-capacity millimetre-wave communications with orbital angular momentum multiplexing," Nature Commun., vol. 5, no. 1, p. 4876, Sep. 2014.

[3] A. Al-Jarro, C. G. Biris, and N. C. Panoiu, "Resonant mixing of optical orbital and spin angular momentum by using chiral silicon nanosphere clusters," Opt. Exp., vol. 24, no. 7, pp. 6945-6958, Apr. 2016.

[4] C. G. Biris and N. C. Panoiu, "Nonlinear surface-plasmon whisperinggallery modes in metallic nanowire cavities," Phys. Rev. Lett., vol. 111, no. 20, Nov. 2013, Art. no. 203903.

[5] M. L. N. Chen, L. J. Jiang, and W. E. I. Sha, "Ultrathin complementary metasurface for orbital angular momentum generation at microwave frequencies," IEEE Trans. Antennas Propag., vol. 65, no. 1, pp. 396-400, Jan. 2017.

[6] L.-J. Yang, S. Sun, and W. E. I. Sha, "Ultrawideband reflectiontype metasurface for generating integer and fractional orbital angular momentum," IEEE Trans. Antennas Propag., vol. 68, no. 3, pp. 2166-2175, Mar. 2020.

[7] B. Thidé et al., "Utilization of photon orbital angular momentum in the low-frequency radio domain," Phys. Rev. Lett., vol. 99, no. 8, Aug. 2007, Art. no. 087701.

[8] F. Tamburini, E. Mari, A. Sponselli, B. Thidé, A. Bianchini, and F. Romanato, "Encoding many channels on the same frequency through radio vorticity: First experimental test," New J. Phys., vol. 14, no. 3, Mar. 2012, Art. no. 033001.

[9] C. Deng, K. Zhang, and Z. Feng, "Generating and measuring tunable orbital angular momentum radio beams with digital control method," IEEE Trans. Antennas Propag., vol. 65, no. 2, pp. 899-902, Feb. 2017.

[10] Z. Zhang, S. Xiao, Y. Li, and B.-Z. Wang, "A circularly polarized multimode patch antenna for the generation of multiple orbital angular momentum modes," IEEE Antennas Wireless Propag. Lett., vol. 16, Jul. 2017, Art. no. 16749438

[11] W. Zhang et al., "Mode division multiplexing communication using microwave orbital angular momentum: An experimental study," IEEE Trans. Wireless Commun., vol. 16, no. 2, pp. 1308-1318, Feb. 2017.

[12] N. Yu and F. Capasso, "Flat optics with designer metasurfaces," Nature Mater., vol. 13, no. 2, pp. 139-150, Feb. 2014.

[13] W. J. Byun et al., "Multiplexed Cassegrain reflector antenna for simultaneous generation of three orbital angular momentum (OAM) modes," Sci. Rep., vol. 6, no. 1, p. 27339, Jun. 2016.

[14] K. Liu et al., "Generation of OAM beams using phased array in the microwave band," IEEE Trans. Antennas Propag., vol. 64, no. 9, pp. 3850-3857, Sep. 2016.

[15] Y. Gong, R. Wang, B. Zhang, N. Wang, N. Li, P. Wang, and Y. Deng, "Generation and transmission of OAM-carrying vortex beams using circular antenna array," IEEE Trans. Antennas Propag., vol. 65, no. 6, pp. 2940-2949, Jun. 2017.
[16] X. D. Bai et al., "Experimental array for generating dual circularlypolarized dual-mode OAM radio beams," Sci. Rep., vol. 7, Jan. 2017, Art. no. 40099.

[17] M. Farmahini-Farahani and H. Mosallaei, "Birefringent reflectarray metasurface for beam engineering in infrared," Opt. Lett., vol. 38, no. 4 , pp. 462-464, Feb. 2013.

[18] R. Florencio, J. A. Encinar, R. R. Boix, M. Barba, and G. Toso, "Flat reflectarray that generates adjacent beams by discriminating in dual circular polarization," IEEE Trans. Antennas Propag., vol. 67, no. 6, pp. 3733-3742, Jun. 2019.

[19] G.-B. Wu, S.-W. Qu, S. Yang, and C. H. Chan, "Broadband, single-layer dual circularly polarized reflectarrays with linearly polarized feed," IEEE Trans. Antennas Propag., vol. 64, no. 10, pp. 4235-4241, Oct. 2016.

[20] R. Elsharkawy et al., "Single-and double-beam reflectarrays for Ka band communication," Sādhanā, vol. 44, no. 5, pp. 1-13, Apr. 2019.

[21] H.-F. Huang and S.-N. Li, "Single-layer dual-frequency unit for multifunction OAM reflectarray applications at the microwave range," Opt. Lett., vol. 45, no. 18, pp. 5165-5168, Sep. 2020.

[22] H.-F. Huang and S.-N. Li, "High-efficiency planar reflectarray with small-size for OAM generation at microwave range," IEEE Antennas Wireless Propag. Lett., vol. 18, no. 3, pp. 432-436, Mar. 2019.

[23] Z. H. Jiang, Y. Zhang, and W. Hong, "Anisotropic impedance surfaceenabled low-profile broadband dual-circularly polarized multibeam reflectarrays for Ka-band applications," IEEE Trans. Antennas Propag., vol. 68, no. 8, pp. 6441-6446, Aug. 2020.

[24] X. Meng, J. Wu, Z. Wu, T. Qu, and L. Yang, "Dual-polarized reflectarray for generating dual beams with two different orbital angular momentum modes based on independent feeds in C-and X-bands," Opt. Exp., vol. 26, no. 18, pp. 23185-23195, Sep. 2018.

[25] K. Zhang et al., "Phase-engineered metalenses to generate converging and non-diffractive vortex beam carrying orbital angular momentum in microwave region," Opt. Exp., vol. 26, no. 2, pp. 1351-1360, Jan. 2018.

[26] J. Yang et al., "Generation of radio vortex beams with designable polarization using anisotropic frequency selective surface," Appl. Phys. Lett., vol. 112, no. 20, May 2018, Art. no. 203501.

[27] Y. Meng, J. Yi, S. N. Burokur, L. Kang, H. Zhang, and D. H. Werner "Phase-modulation based transmitarray convergence lens for vortex wave carrying orbital angular momentum," Opt. Exp., vol. 26, no. 17, pp. 22019-22029, Aug. 2018.

[28] H. Ahmed, A. A. Rahim, H. Maab, M. M. Ali, and S. Naureen, "Polarization insensitive all-dielectric metasurfaces for the ultraviolet domain," Opt. Mater. Exp., vol. 10, no. 4, pp. 1083-1091, Apr. 2020.

[29] W. Wan, J. Gao, and X. Yang, "Full-color plasmonic metasurface holograms," ACS Nano, vol. 10, no. 12, pp. 10671-10680, Dec. 2016.

[30] H. Cheng et al., "Dynamically tunable broadband infrared anomalous refraction based on graphene metasurfaces," Adv. Opt. Mater., vol. 3, no. 12, pp. 1744-1749, Dec. 2015.

[31] L. Ye, H. Feng, G. Cai, Y. Zhang, B. Yan, and Q. H. Liu, "High-efficient and low-coupling spoof surface plasmon polaritons enabled by V-shaped microstrips," Opt. Exp., vol. 27, no. 16, pp. 22088-22099, Aug. 2019.

[32] S. Liu et al., "Anisotropic coding metamaterials and their powerful manipulation of differently polarized terahertz waves," Light, Sci. Appl., vol. 5, no. 5, Jan. 2016, Art. no. e16076.

[33] Y. Ling et al., "Polarization-controlled dynamically switchable plasmoninduced transparency in plasmonic metamaterial," Nanoscale, vol. 10, no. 41, pp. 19517-19523, Sep. 2018.

[34] B. Sima, K. Chen, X. Luo, J. Zhao, and Y. Feng, "Combining frequencyselective scattering and specular reflection through phase-dispersion tailoring of a metasurface," Phys. Rev. Appl., vol. 10, no. 6, Dec. 2018, Art. no. 064043.

[35] A. Arbabi, Y. Horie, M. Bagheri, and A. Faraon, "Dielectric metasurfaces for complete control of phase and polarization with subwavelength spatial resolution and high transmission," Nature Nanotechnol., vol. 10, no. 11, pp. 937-943, Aug. 2015.

[36] Y. Zhang, Y. Feng, T. Jiang, J. Cao, J. Zhao, and B. Zhu, "Tunable broadband polarization rotator in terahertz frequency based on graphene metamaterial," Carbon, vol. 133, pp. 170-175, Jul. 2018.

[37] S. Mercader-Pellicer, G. Goussetis, G. M. Medero, H. Legay, D. Bresciani, and N. J. G. Fonseca, "Cross-polarization reduction of Linear-to-Circular polarizing reflective surfaces," IEEE Antennas Wireless Propag. Lett., vol. 18, no. 7, pp. 1527-1531, Jul. 2019.

[38] N. Yu et al., "Light propagation with phase discontinuities: Generalized laws of reflection and refraction," Science, vol. 334, no. 6054, pp. 333-337, Oct. 2011. 
[39] C.-F. Chen, C.-T. Ku, Y.-H. Tai, P.-K. Wei, H.-N. Lin, and C.-B. Huang, "Creating optical near-field orbital angular momentum in a gold metasurface," Nano Lett., vol. 15, no. 4, pp. 2746-2750, Mar. 2015.

[40] F. Yue et al., "Multichannel polarization-controllable superpositions of orbital angular momentum states," Adv. Mater., vol. 29, no. 15, Apr. 2017, Art. no. 1603838 .

[41] B. Berger, M. Kahlert, D. Schmidt, and M. Assmann, "Spectroscopy of fractional orbital angular momentum states," Opt. Exp., vol. 26, no. 24, pp. 32248-32258, Nov. 2018.

[42] G. Ding, K. Chen, X. Luo, J. Zhao, T. Jiang, and Y. Feng, "Dual-helicity decoupled coding metasurface for independent spin-to-orbital angular momentum conversion," Phys. Rev. Appl., vol. 11, no. 4, Apr. 2019, Art. no. 044043

[43] H. Shi et al., "Generation of multiple modes microwave vortex beams using active metasurface," IEEE Antennas Wireless Propag. Lett., vol. 18, no. 1, pp. 59-63, Jan. 2019.

[44] S. Yu, L. Li, G. Shi, C. Zhu, X. Zhou, and Y. Shi, "Design, fabrication, and measurement of reflective metasurface for orbital angular momentum vortex wave in radio frequency domain," Appl. Phys. Lett., vol. 108, no. 12, Mar. 2016, Art. no. 121903.

[45] G. Ding, K. Chen, T. Jiang, B. Sima, J. Zhao, and Y. Feng, "Full control of conical beam carrying orbital angular momentum by reflective metasurface," Opt. Exp., vol. 26, no. 16, pp. 20990-21002, Aug. 2018.

[46] W.-L. Guo et al., "Broadband polarization-conversion metasurface for a cassegrain antenna with high polarization purity," Phys. Rev. Appl., vol. 12, no. 1, Jul. 2019, Art. no. 014009.

[47] M. I. Khan, Z. Khalid, and F. A. Tahir, "Linear and circular-polarization conversion in X-band using anisotropic metasurface," Sci. Rep., vol. 9, no. 1, p. 4552, Mar. 2019.

[48] J. Lončar, A. Grbic, and S. Hrabar, "A reflective polarization converting metasurface at X-band frequencies," IEEE Trans. Antennas Propag., vol. 66, no. 6, pp. 3213-3218, Jun. 2018.

[49] L. Marrucci, C. Manzo, and D. Paparo, "Optical spin-to-orbital angular momentum conversion in inhomogeneous anisotropic media," Phys. Rev. Lett., vol. 96, no. 16, p. 163905, Apr. 2006.

[50] M. Q. Mehmood et al., "Visible-frequency metasurface for structuring and spatially multiplexing optical vortices," Adv. Mater, vol. 28, no. 13, pp. 2533-2539, Apr. 2016.

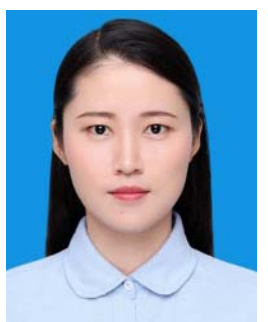

Fengxia $\mathbf{L i}$ received the B.S. degree in electronic information science and technology from Henan Polytechnic University, Jiaozuo, China, in 2015. She is currently pursuing the Ph.D. degree in microelectronics and solid states electronics at the University of Electronic Science and Technology of China, Chengdu, China.

Her recent research interests include the structural design of novel metamaterials and their applications in electromagnetic wave modulation, frequency-selective surfaces, radar cross section reduction, and wave absorbers.

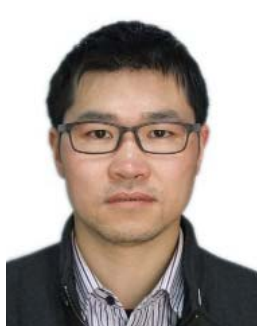

Haiyan Chen received the Ph.D. degree in microelectronics and solid states electronics from the University of Electronic Science and Technology of China (UESTC), Chengdu, China, in 2011.

In 2015, he joined the Department of Engineering, University of Kentucky, Lexington, KY, USA, as a Visiting Scholar. Since 2012, he has been with the National Engineering Research Center of Electromagnetic Radiation Control Materials, UESTC, where he is currently a Professor. His current research interests include artificial electromagnetic (EM) material and EM radiation control materials, particularly study on EM discontinuous repair materials.

Dr. Chen was a recipient of the Chinese Scholarship Council Scholarship in 2015 .

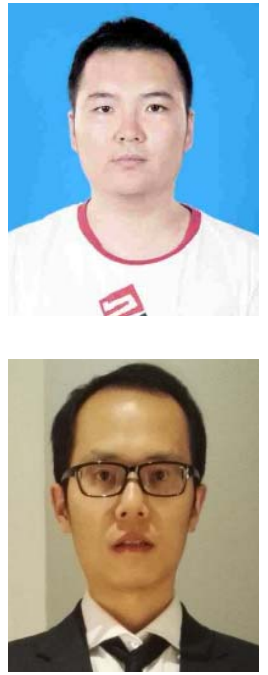

Yang Zhou was born in Liaoning, China, in 1989. He received the B.S. degree in electronic science and technology and from the University of Electronic Science and Technology of China, Chengdu, China, in 2012 and 2019, respectively.

His recent research activities have focused on the numerical modeling of novel metamaterials and their applications in wave modulation, antenna arrays, and wave absorbers.

Research Associate His rese netics, nonlinear microwave and optics, quantum metamaterials, multiphysics, and computational plasma physics.

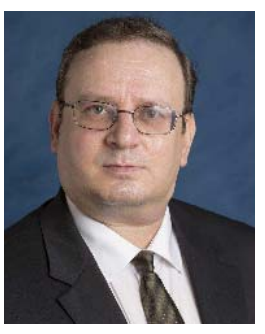

Nicolae C. Panoiu (Member, IEEE) received the B.Sc. and M.S. degrees in physics from the University of Bucharest, Bucharest, Romania, in 1990 and 1992, respectively, and the Ph.D. degree from New York University (NYU), New York, NY, USA, in 2001.

After graduating from NYU, he was a Post-Doctoral Fellow with the Department of Applied Physics and Applied Mathematics, Columbia University, New York. He is currently a Professor of nanophotonics with the Department of Electronic and Electrical Engineering, University College London, London, U.K. His research interests include silicon photonics, optical properties of photonic nanostructures and metamaterials, and computational modeling of electromagnetic structures.

Dr. Panoiu is a member of The Optical Society.

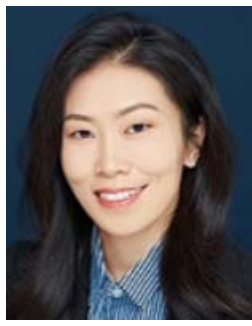

Peiheng Zhou (Member, IEEE) received the Ph.D. degree in material physics and chemistry from the University of Electronic Science and Technology of China (UESTC), Chengdu, China, in 2009.

She has been with the State Key Laboratory of Electronic Thin Films and Integrated Devices and the National Engineering Research Center of Electromagnetic Radiation Control Materials, UESTC, where she is currently a Full Professor. From November 2007 to November 2008, she was a Research Visitor with the Massachusetts Institute of Technology, Cambridge, MA, USA. From September 2018 to April 2020, she was a Research Visitor with Nanyang Technological University, Singapore. Her current research interest includes various aspects of electromagnetic materials or structures, particularly the interplay between electromagnetic wave and materials.

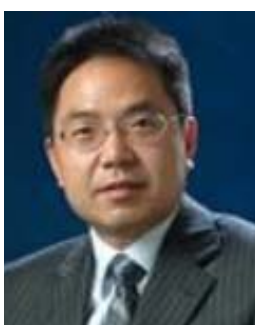

Longjiang Deng received the M.S. degree in electronic material and device from the University of Electronic Science and Technology of China (UESTC), Chengdu, China, in 1987.

Since 1987, he has been working with UESTC, as a Lecturer, an Associate Professor, and a Full Professor. He has authored or coauthored about 200 articles in refereed international journals and industry publications and given many invited talks in international conferences. His research interests include electromagnetic wave absorbing material, infrared low emissivity and selective emissivity thin film, and microwave magnetic material.

Mr. Deng is a member of the Branch of the Chinese Institute of Electronics on Microwave Magnetism, the Editor Committee of the Chinese Journal of Functional Materials, and the Vice Director of the Special Committee of the Chinese Institute of Electromagnetic Material. 